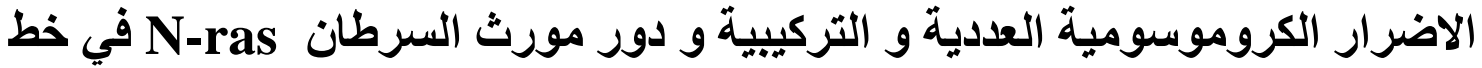

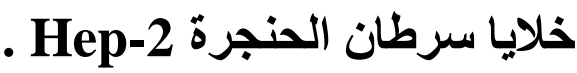

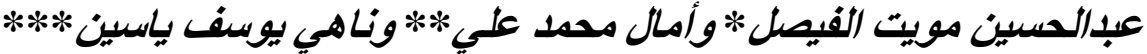

\author{
2008/5/27 ت تاريخ قبول النشر
}

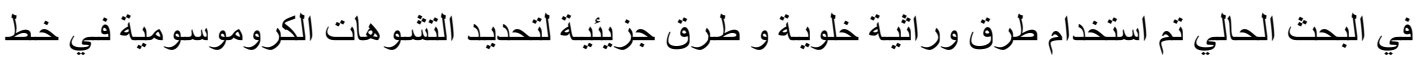

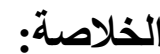

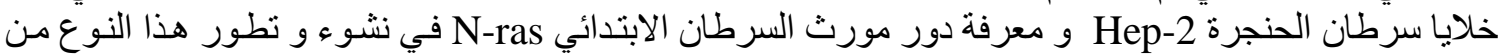

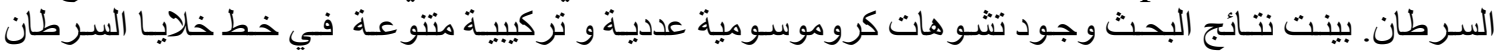

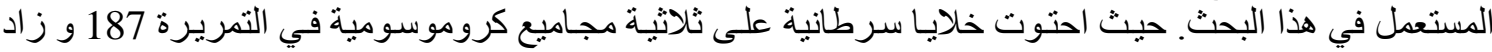

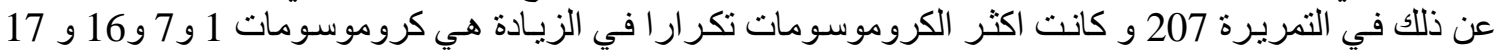

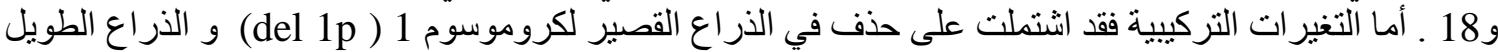
لكروموسومي 6 (del 6q) و كروموسوم 1 (del 1q) ـ كما سجل وجود زيادة في الذر اع القصير لكروموسومي 3

أما على المستوى الجزيئي فقد تبين من خلال استخدام تقنية تفاعل سلسلة البوليمريز PCR و و بادئات لموقعين و 12.

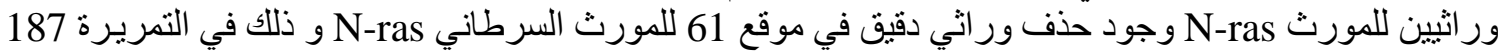

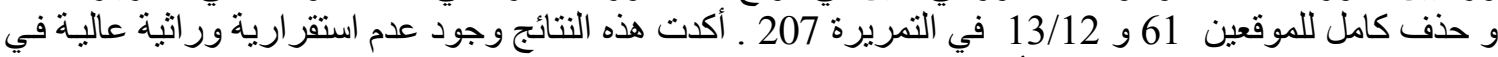

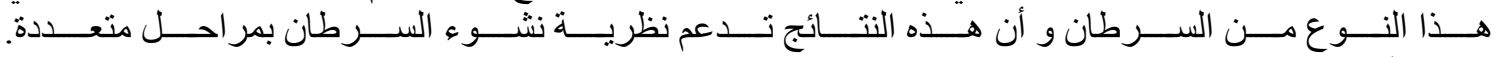

يمكن مشاهدة عدم استقر ارية المحتوى الوراثي هذا

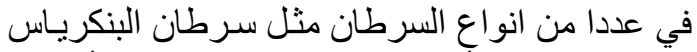

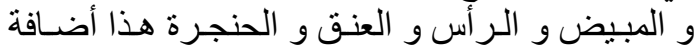

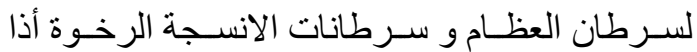

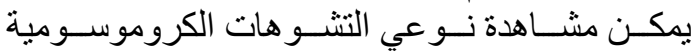

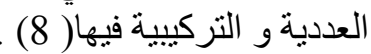

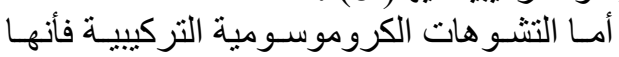

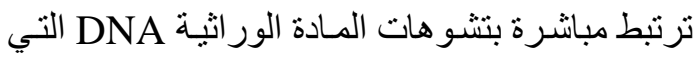

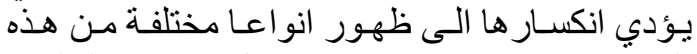

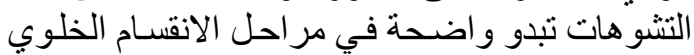

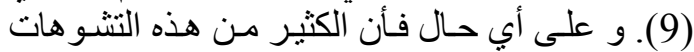

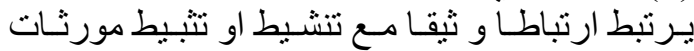
سرطانية ابتدائية C-Oncogenes او مورثنات كابتـة للاورام (12,11,10). Suppressor genes. أن افضل الوسائل و الانظمـة المناسبة لتحقيق

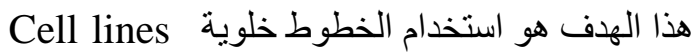

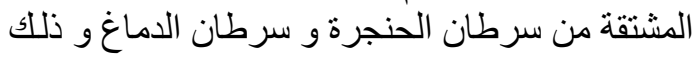

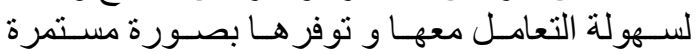

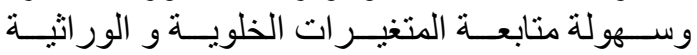

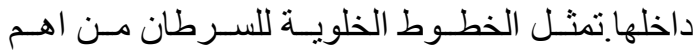

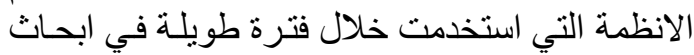

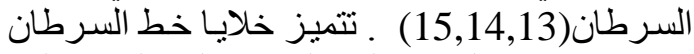

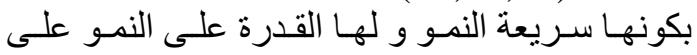

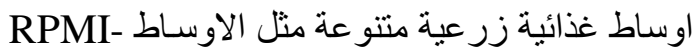

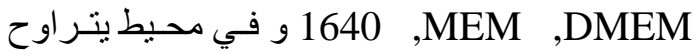

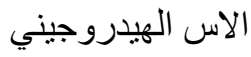

المقدمة : الميز

يتميز السرطان بعدم قدرتة على الاحتفاظ على

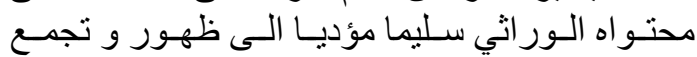

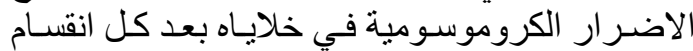

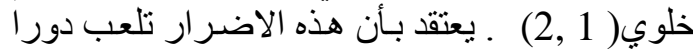

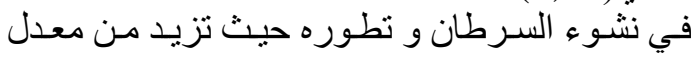

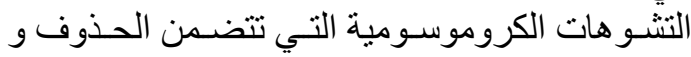

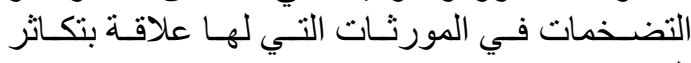

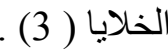

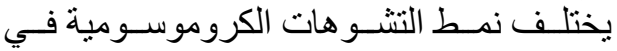

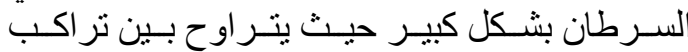

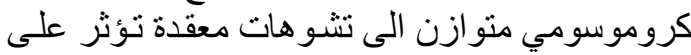

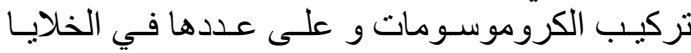

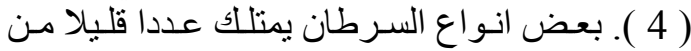

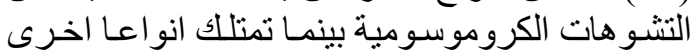

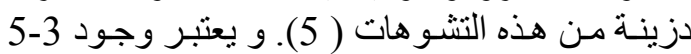

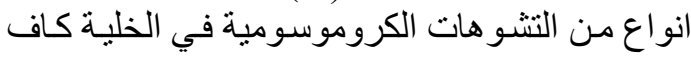

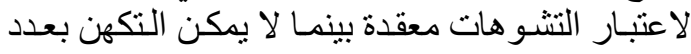

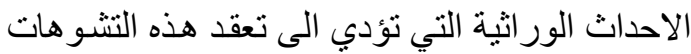

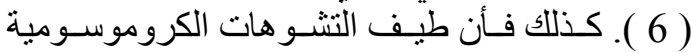

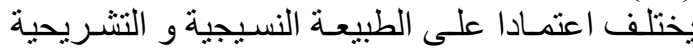

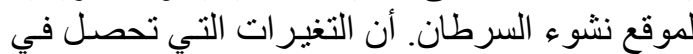
كروموسومات الخلايا السرطانية تعكس وجود النير اليات التيات

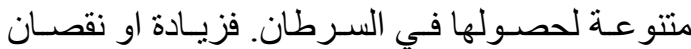

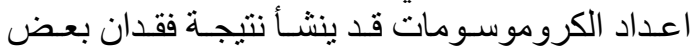

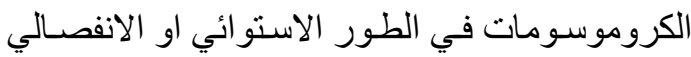

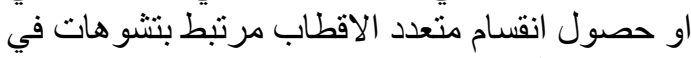

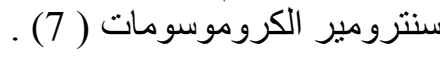

*دكتوراه في الوراثة الطبية الجزيئية /معهد الهندسة الوراثية و التقنيات

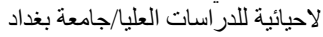

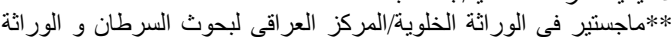

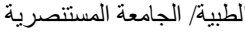

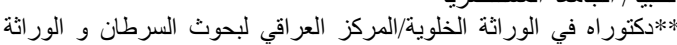

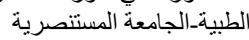




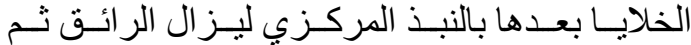
اضيف5 مل من المثبت (3أجز اء من كحول الايثانول

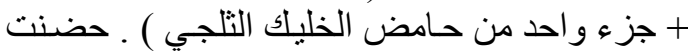

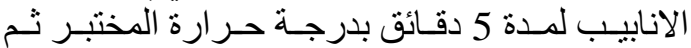

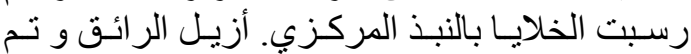

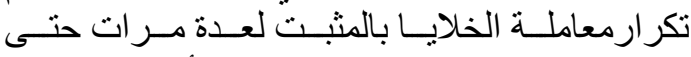

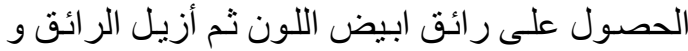

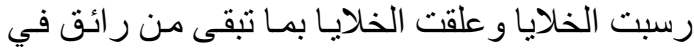
الانابيب.

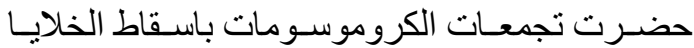

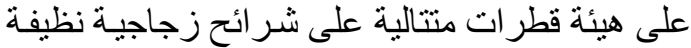

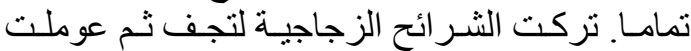

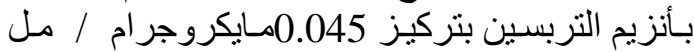

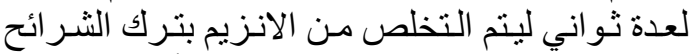

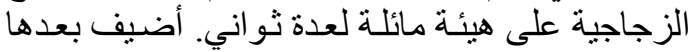

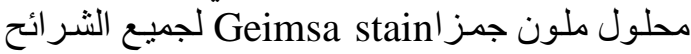
الزجاجية و تركت الثرائح مع الملون لمدة 3-2دقيقة

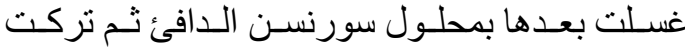
لتجف.

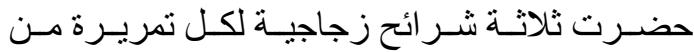

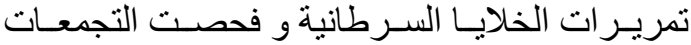

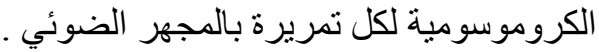

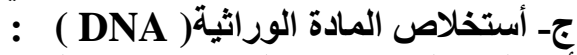

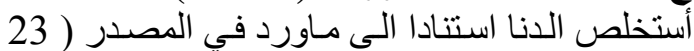

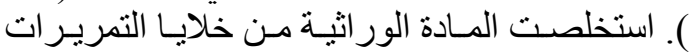

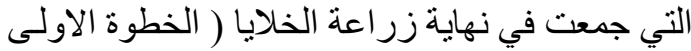

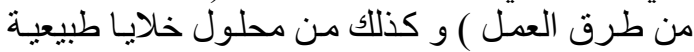

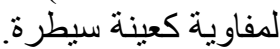

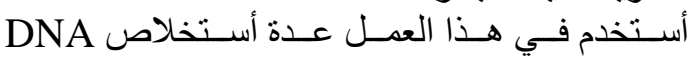

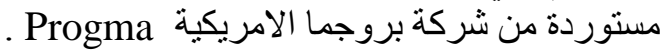

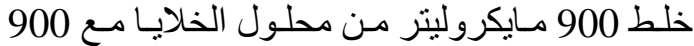
مايكروليتر من محلول تحلل الخلايا في انابيب نظيفة فئسة

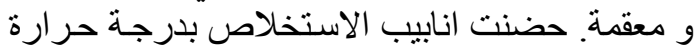

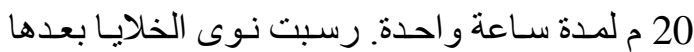
بالنبذ المركزي بسر عة 3000 دورة في الدقيقة. أزيل

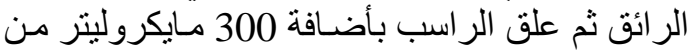

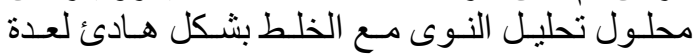

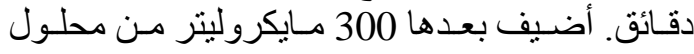

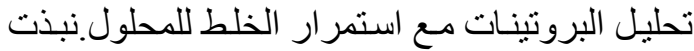

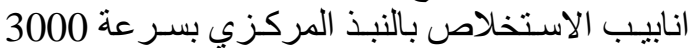
دورة في الدقيقة لمدة 10 دقائق ثم نقل الرئر ائق الذية الذي

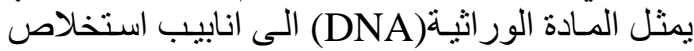

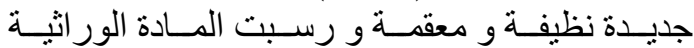

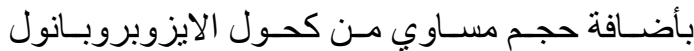

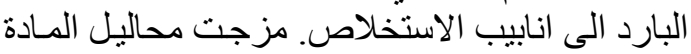

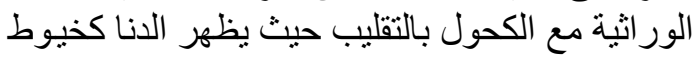

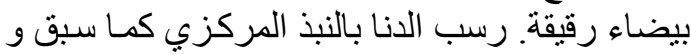

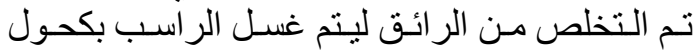

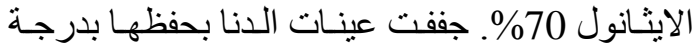

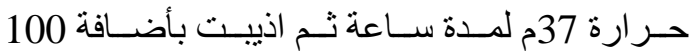
مايكروليتر من الماء المقطر.

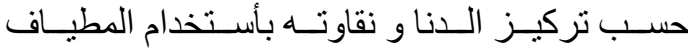
الضـوئي عند طول مـوجي 260 و 280 انكستروم. النسطياف

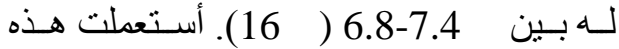
الخطوط الخلوية في العديد من الابحاث التجريبية مثل التل

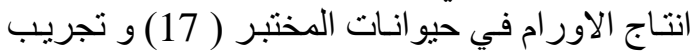

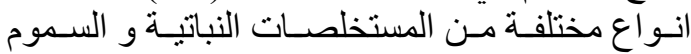
البكتيرية ( 18) و در اسـات التغيرات الكروموسومية التية التهات

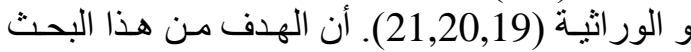

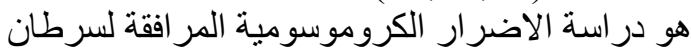
الحنجرة و كذللك دراسة دور مورث الكرو السرومة السرطان الاولي N-ras

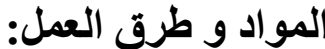
أ ـ ـ تهيئة الخلايا السرطانية:

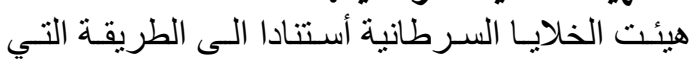

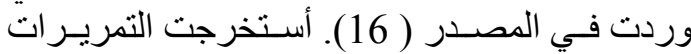

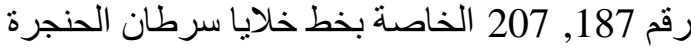

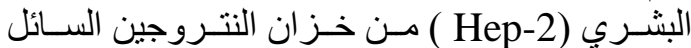

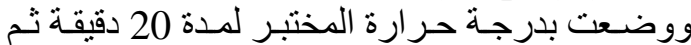

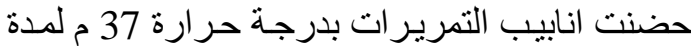
5 دقائق. نقلت محتويات كل انبوبـة الى فتينـة زر راعـة

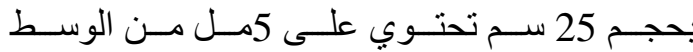
الغذائيمPMI-1640 المقوى ب 10\%مـن مصـل

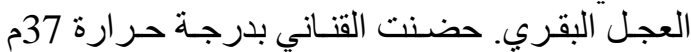
داخل حاضنة مزودة ب $5 \%$ ثناني اوكسيد الكاربون

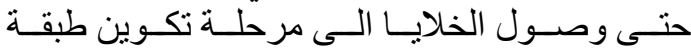
خلويـة conflent مع مر اعـاة تغيير الوسط الغذائي

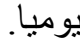

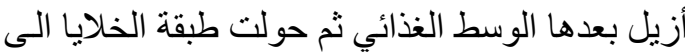

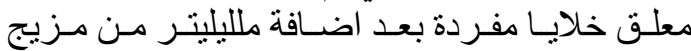

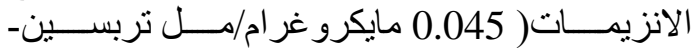

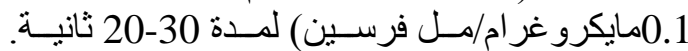
وبأستخدام 2-3 مل من الوسط الغذائي نم شفط الخلايايا

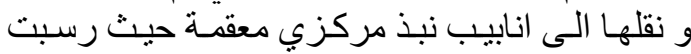

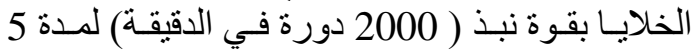

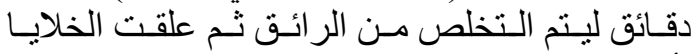

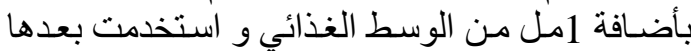

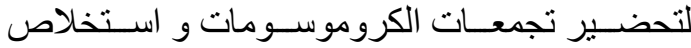
المادة الور اثية من كل تمريرة.

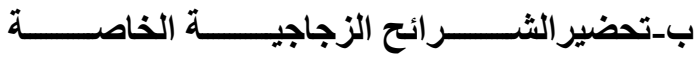

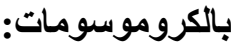
حضرت تجمعات الكروموسومات استنادا الى ما ورد

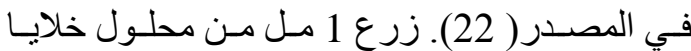

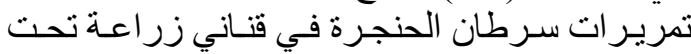

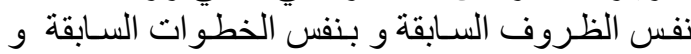

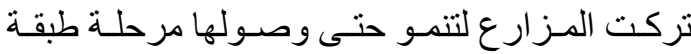

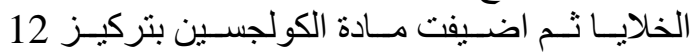

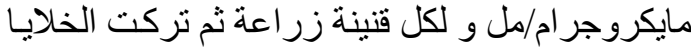
مع هذه المادة لمدة 30-20دوقيقة.

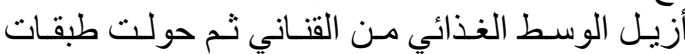

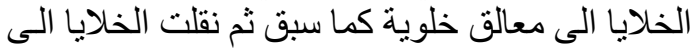

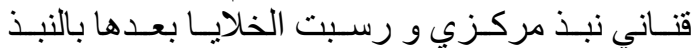
المركزي بنفس الظروف السابقة. علق ر اسب الخلايـا بما تبقى من وسط غذائي ثم أضيف 3 مل من محلون

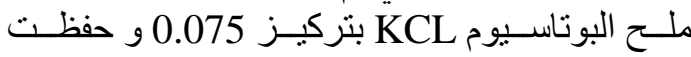
الانابيب بدرجـة حرارة 37 م لمدة 30 دقيقة. رسبت 
تراوح العدد الكلي للكروموسومات في خط خلايا

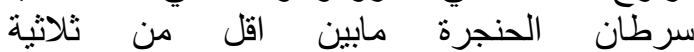
المجموعة|الكروموسومية في التمريرة 187 و و اكثر

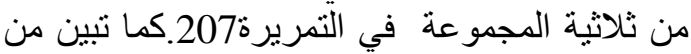

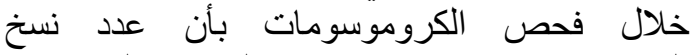

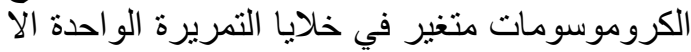

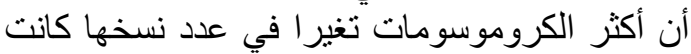

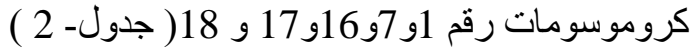
جدول2-2 : النغيرات العددية المسلة في تمريرات خط خلايا سرطان

\begin{tabular}{|c|c|c|c|}
\hline الكروموسومى الدمى & الننوال & الخلاديا & التنريرية \\
\hline $50-72$ & 65 & 35 & 187 \\
\hline $63-83$ & 76 & 21 & 207 \\
\hline
\end{tabular}

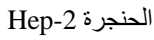

أما التغيرات التركيبية فقد شملت فقدان جزئي او كلي

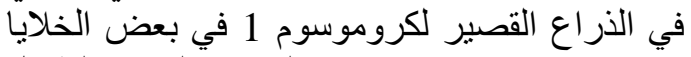

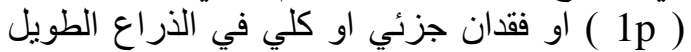

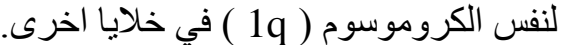
كما شملت التغيرات زيادة جزئية او كلية في الذيا لفرواع

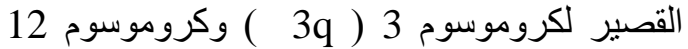

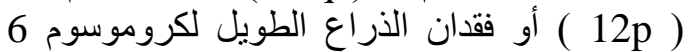

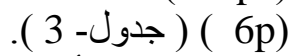

ويجدر الذكر بأن التغيرات التركيبية الانفة الذكر

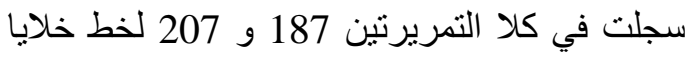
سرطان ألحنجرة الا انها كانت اكثر استقرارا في

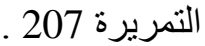

جدول 3:التغيرات التركيبية التي سجلت في خط خلايا سرطان الحنجرة Hep-2.

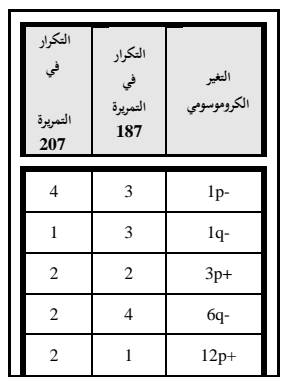

بـ دور مورث السرطان N-ras في سرطان

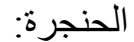

يوضح شكل -1 نتائج تحليل الدنا المستخلص من المناع تمريرات مختلفة لسرطان الحنجرة و بأستخدام تفاعل

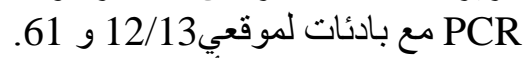

يظهر من خلال الثنكل بأن هناك فقدان في الموقع 61 لمورث السرطان الابتدائي N-ras في التمريرة في لمان 187 لخط سرطان الحنجرة Hep-2 حيث الظهر تفاعل وجود حزمة واحدة بحجم) PCR الموقع 13/12 فيما اختفت كلا الحزمنين في التمريرة 207 مقارنة مع العينة الطبيعية.

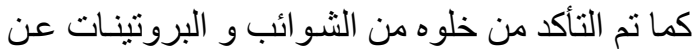

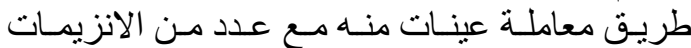

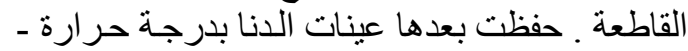
20م حتى استخدامها في تفاعلات سلسلة البوليميريز

.Polymerase chain reaction-PCR لــ تفاعل سلسلة البوليميريز) (PCR

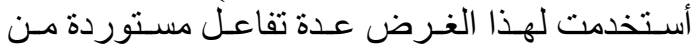

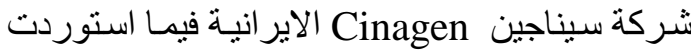

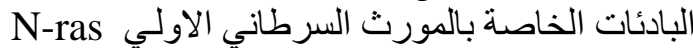

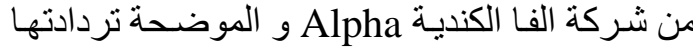

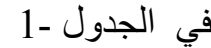

جدول - 1: ترددات البادئات المستخدمة في تفاعل سلسلة البوليميريز PCR.

\begin{tabular}{|c|c|c|c|}
\hline لثرى & البادة|ة الامائية & البادةرة الماكستة & السرطاين \\
\hline $12 / 13$ & $\begin{array}{c}\text { 5- } \\
\text { ATGACTGAGTACAACTGGT } \\
-3\end{array}$ & $\begin{array}{c}\text { 5- } \\
\text { AATATGATCCCACATAGAG - } \\
3\end{array}$ & N-ras \\
\hline 61 & $\begin{array}{c}5 \\
\text { CAAGTGGTTATAGATGGTGA } \\
-3\end{array}$ & $\begin{array}{c}\text { 5- } \\
\text { AGGACAGGGAAGGCTTCCTC } \\
-3\end{array}$ & N-ras \\
\hline
\end{tabular}

اجري تفاعل سلسلة البوليميريز PCR اعتمادا على ديك

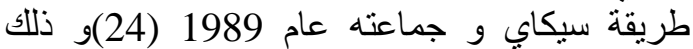
بأستخدام جهاز دورات تفاعل PCR من شركة

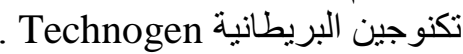

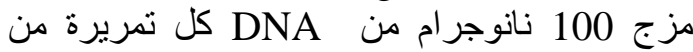
تمريرات كل نوع من السرطان مع 35 مايكروليتر تيرير Master من محلول تفاعل سلسلة البوليميريز

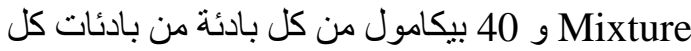

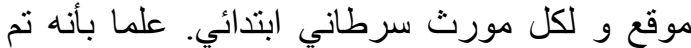

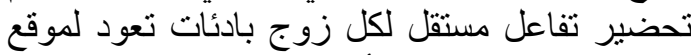
واحد و لمورث و واحد. أكمل حجم التفاعل باعل التى 50 مايكروليتر بأضافة ماء مقطر معق ثم

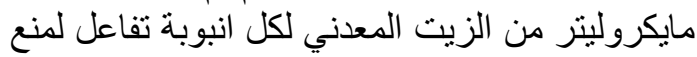
تبخر محتويات التفاعل. وضنعت الانابيب في جهاز دورات تفاعل PCR وفق البرنامج المناسب للتنفاعل (95)) لمدة 20 ثانية و 72 درجة مئوية لمدة دئة 30 ثانية و لغاية دئة 20 دورة تفاعلية ) .

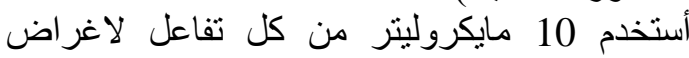
الهجرة الكهربائية عبر هلام الاجاروز بتركيز 10 1

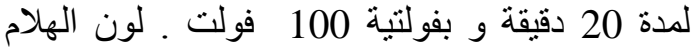

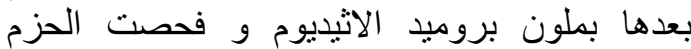
الناتجة من التفاعل بجهاز الاشعة فوق البنفسجية ثم

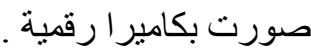

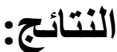
أ-التغيرات الكروموسومية العددية و التركيبية في خلايا الخطوط السرطانية: تضمن العمل دراسة هذه التغيرات في التمريرنان

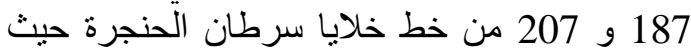
فحص ما معدله 50 خلية في الطور الاستو ائي لكل

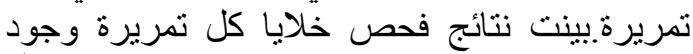

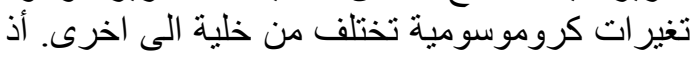




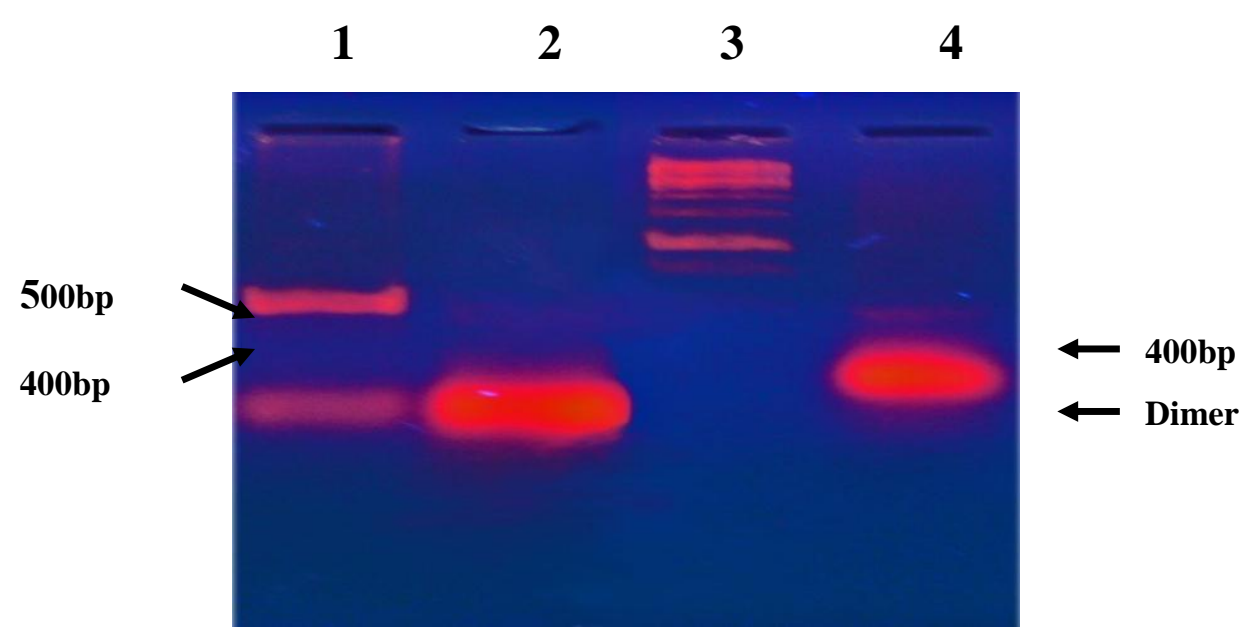

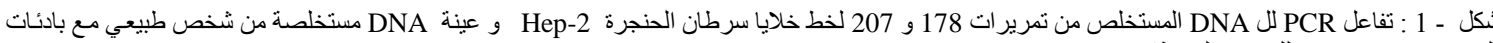

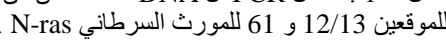

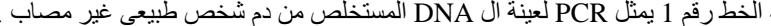

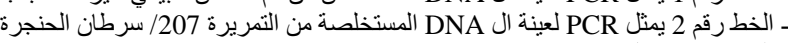

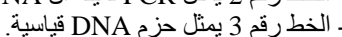

هناك حذف كامل لمورث - الخطر رقم 4 يمثل يمثل حزم لعينة ال DNA المستخلصة من التمريرة 187 / سرطان الحنجرة.

12/13و 61 في التمريرة 207 لخط سرطان في فئرن

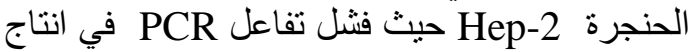

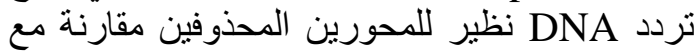

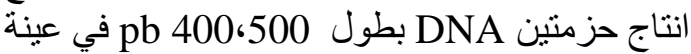

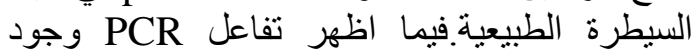
حذف في الموقع 61 في DNA التمريرة 187 لخط

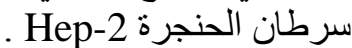

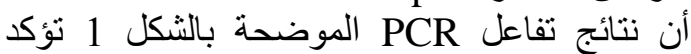

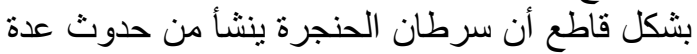
تغيرات وراثية متتابعة يمثل الحذف في الموني

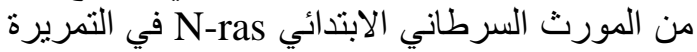

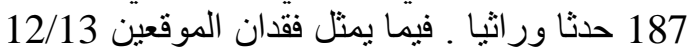
و 61 لنفس المورث في التمريرة 207 حدثا ور اثثانيا اخر هذا اضافة للتثنو هات الكروموسومية المسجلة. أن أن

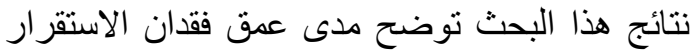

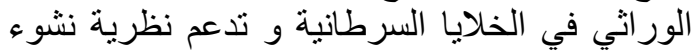
السرطان نتيجة حصول احداث ور راثية عديدة متعاقبة. أن هذه النتائج تتطابق مع ما وجناث التهاه في الفحوصات

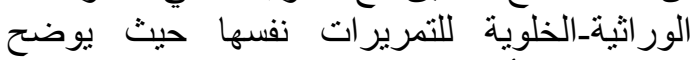

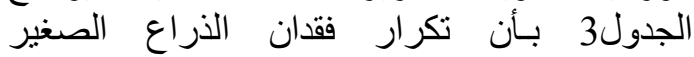

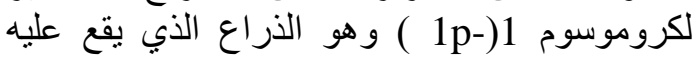

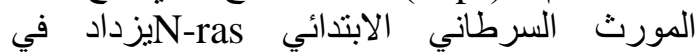
التمريرة 207 مقارنة مع التمريرة 178 لـونئن

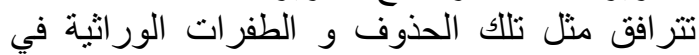

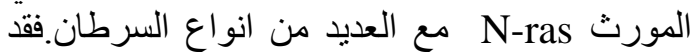

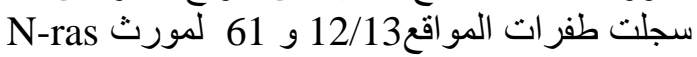
في ابحاث اخرى حول سرطان الحنجرة ( 42,41 ).

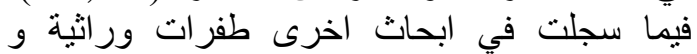

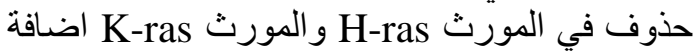
لطفرات وراثية في المورث N-ras في سرطان

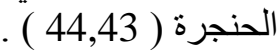
كما سجلت مثل تلك التغيرات الوراثية في المورث

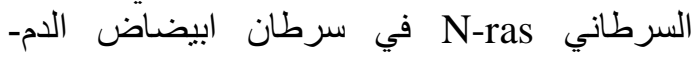

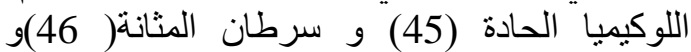

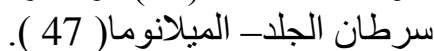

\section{المناقثة :} بينت نتائج الفحوصات الكروموسومية التي اجريت

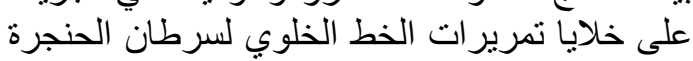

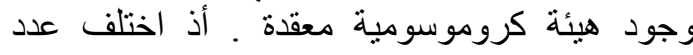

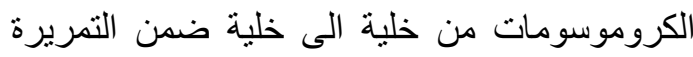

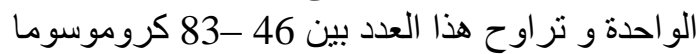

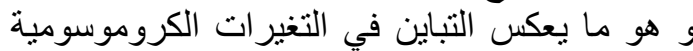

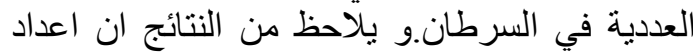

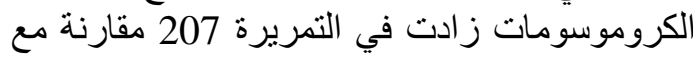

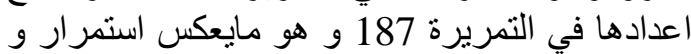
تز ايد حصول التنغير ات الور اثية في الخلايا السرطانية مع تقدها بالعمر.. أتخلف أعداد نسخخ الكروموسومات في خلايا كلتا

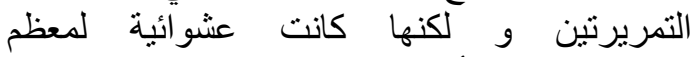

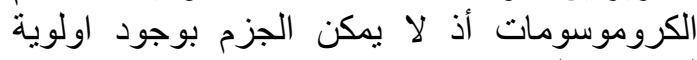

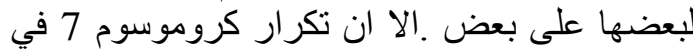
دراسات مماتلة اوضح تغيرا غير عشو ائي (16).وقد

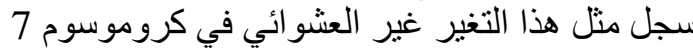

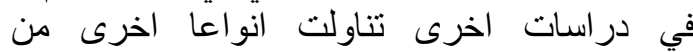
السرطان ( 26,25).فقد سجلت انواع من التغ التغيرات

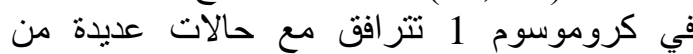

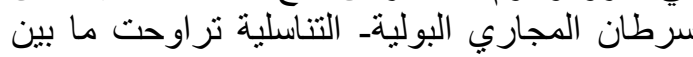

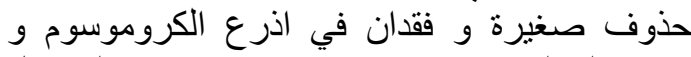

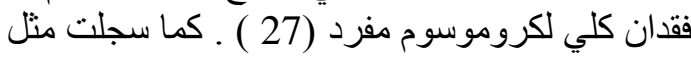
هذه التغيرات في كروموسوم 1 في سرطان الغدان الصرماء ( 28 ) )و سرطان المعدة (29) و فروس سرطان

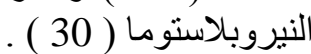
كما سجلت انو اع مختلفة من التغير ات الكروموسومية و الجينية في حالات من انواع مختلفة من سرطان الكرونان

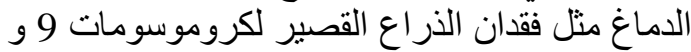

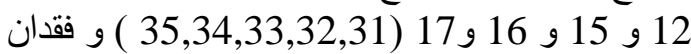
الذراع الطويل لكروموسومات 6 و 13 و و 16 و و19 و و 22 (37,36 ) و وقدان كامل لكروموسومات 10 و 10 و 22 (40,39,38 ) . توضح نتائج الثنكل-1 بأن 
Journal of Clinical Oncology5:16101979.

10- Sanchez-Barcelo,E.J,Cos,S.,ernanez, and Mediavilla,M.D.2003.Endorinrelated cancer.Cancer 10:153-159

11- 11.Stites,E.C.,Paul,C.T.,Zhong,M., Kodi,S.R.2007.Network Analysis of Oncogenic Ras Activation in Cancer .Science 318:463-467.

12- Myers,M.E.,Chang,M.H.,Jorgensen, C.,Whitworth,W.,Kassim,S.,Litch,J. A.,Armstrong,L.,Bernhardt,B.,Faucet t,W.A.,Irwin,D.,Mouchawar,J.and Bradley,L.A.2006.Genetic testing for susceptibility to breast and ovarian cancer;Evaluating the impact of a direct to consumer marketing campaign on physicians knowledge and practices.Genetic Med.8:361-370.

13- Pirhonen' J.,Timo,S.,Masashi,K., Ilkka,J.and Sampsa M.1999.Virus Infection Activates IL-1ß and IL-18 Production in Human Macrophages by a Caspase-1-Dependent Pathway. The Journal of Immunology,162: 7322-7329

14- Beutin,L.,Olivier,M.,Karl,A.B.,Ker stin,G.,Sonja,Z.,Herbert,S.and Eric O.2003.HEp-2Cell Adherence,Actin Aggregation, and Intimin Types of Attaching and Effacing Escherichia coli Strains Isolated from Healthy Infants in Germany and Australia.Infect Immun.71(7):3995-4002.

15- Li,Yinghui;Li,F.,Li-Ling,J.,Wang,X .,Xu,Z.andSun,K.2005.STK15Gene Overexpression,Centrosomal Amplification, and Chromosomal Instability in the Absence of STK15 Mutations in Laryngeal Carcinoma. Cancer Investigation,23(8):660-664.

16- Ali,A.M.2004.Cytogentic and immunological study on larynx carcinoma cell line.MSc.Thesis,Baghdad University-College of science.

17- Notter,M.F.D.and Docherty,J.J. 2005. Steroid hormone alteration of herpes simplex virus type 1 replication.Journal of Medical Virology

18- Ozawa,T.,Nishikimi,M.,Sugiyama,S .,Taki,F.,Hayakawa,M. and Shionoya,H.1988.Cytotoxic activity of leukotoxin,aneutrophil derived fatty

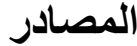

1- Al-Faisal, A.H.M., Al-Katteb,I. and Al-Katteb,F.2000.DNA Ploidy and Heterogeneity in Ovarian Cancer. Cancer Mol. Biol.7:1471 -1479.

2- Al-Faisal,A.H.M.,Al-Katteb,I.and Habashna,M.2001.Elevation of Prostatic Speeific Antigen in Prostate benign hyperplerplasia and prostatic $\mathrm{Ca}$ ncer.Cancer Mol.Biol.8:1591-1597.

3- Larson,P.L.,Schlechter,B.L.,Morenas ,A.D.,Garber,J.E.,Gupples,L.A., and Rosenberg,C.L.2005.Allelr imbalaneor loss of heterozygosity in normal breast epitheliaum of sporadic breast cancer cases and BRCA1 gene mutaion carriers is increased compared with reduction mammoplastyssues J.Clin.ncol.34 8613-8619.

4- Yoon,S.K.,Lim,N.K.,Ha,S.A.,Park,y. G.,Choi,J.Y.Chung,K.W.,Sun,H.S.,C hoi,M.J.,Chung,J.,Wands,J.R.and kim,J.W.2004. Human cervical cancer oncogene protein is a biomaker from human hepatocellular carcnoma. Cancer Res. 64:543-544.

5- Fan,X.,Irina,M.,Ihab,E.,Xing,Z.N., Yunyue,W.,Douglas,B.,Daniel,J.B.,A -ieP.and Charles,G.E.2004.Notch1 and Notch2 Have Opposite Effects on Embryonal Brain Tumor Growth. Cancer Research 64,7787-7793.

6- Hui,A.B.,Kwok-Wai,L.,Xiao-Lu,Y., Wai-Sang,P.and Ho-Keung,N.2001 .Detection of Multiple Gene Amplifications in Glioblastoma Mutiforme Using Array-Based Compcative Genomic Hybridization. Lab Invest. 81:717-723.

7- 7.Colorado,P.C.,Torre,A.,Kamphaus, G.,et al.2000.Anti-angiogeniccues from vascular basement membrane collagen. Cance Res;60:2520-6.

8- Moll ,U.M.and Slade,N.2004.p63 and p73:Roles in Development and Tumor Formation.Mol.Cancer Res.2(7): 371-386.

9- Xiong,C.,KONG,W.,ZHANG,S.and ZHANG,D.2006. Relation between the Expression of K-ras in Hep-2 Cells and Development of Laryngeal Carcinoma.The Chinese-German 
27- Elmula,I.F.2005.Chromosomal changes in uroepithelial carcinomas. Cell \& Chromosome,4:1doi:102(3) :247-252.

28- Ebrahimi,S.A.,Eric,H.W.,Alan,W., Rhona,R.S.,Edward,P.,Jr. and Mark, P.S.1999.Deletion of Chromosome 1 Predicts Prognosis in Pancreatic Endocrine Tumors. Cancer Research 59, 311-315.

29- Chen,Y-J.,Alexander,V.,Zhengping ,Z.,Steve,H.and Robert,T.J.2003 Loss of Heterozygosity of Chromosome 1q in Gastrinomas.Occurrence and Prognostic Significance. Cancer Research 63, 817-823.

30- Ambros,I.M.,Andrea,Z.,Borghild, R.,Gabriele,A.,Ruth,L.,Dieter,P.,Helmut,G.and Peter,F.A.1996.Role of Ploidy,Chromosome 1p, and Schwa$\mathrm{nn}$ Cells in the Maturation of $\mathrm{Ne}$ uroblastoma.New England J.of medicine 334:1505-1511.

31- .Jen,J.,Harper,W.,Bigner,S.H.,Bigne ,D.D.,Papadopoulos,N., Markowitz, S.,Willson,J.K.V,Kinzler,K.W,Vogelstein,B.1994.Deletion of p16 and p15 in brain tumors.Cancer Res $.54: 6353$.

32- Reifenberger,G.,Reifenberger,J.,Ichimura,K.,Melzter,P.S.,Collins,V.P .1994.Amplification of multiple genes from chromosomal region $12 \mathrm{q}$ 13-14 in human malignant gliomas: preliminary mapping of the amplicons shows preferential involvement of CDK4, SAS, and MDM2. Cancer Res.54:4299.

33- Ueki, K.,Rubio,M-P.,Ramesh,V., Correa,K.M.,Rutter,J.L., Von Deimling,A.,Buckler,A.J.,Gusella,J.F.,Louis,D.N.1994 .MTS1/ CDKN2 gene mutations are rare in primary human astrocytomas with allelic loss of chromosome 9p.Hum.Molec. Genet. 3:1841.

34- Rajagopalan,H.\& Lengauer,C.2004 .Progress Aneuploidy and cancer Nature 432: 338-341.

35- Gaiano N,Fishell G.2002.The role of notch in promoting glial and neural stem cell fates.Annu. Rev.Neurosci .25:471-90. acidepoxide on cultured human cells.Biochem.Int. 16:369-373.

19- Wittmer,K.,Klenk,U.,Gdassgen,A., Aust,D.E.,Diebold,j.,Lohrs,U.B. and Baretton,G.B.2000.FISH analysis of gene aberrations(myc,ccna1,erb-B2 , $\mathrm{Rb}$ and $\mathrm{Ar}$ )in advanced prostatic carcinoma befor and after androgen deprivation rherapy. Lab.Invest. 80:1455-1464.

20- Sawer,J.R.,Husain,M.and Al-Mefly, O.2001.Identification of isochromosomes $1 \mathrm{q}$ as a recurring chromosome aberration in skull base chordomas:a new manker for aggressive tumors.Neurosurg.Focus 10:1-16.

21- Lensch,,R.,Gotz,C.,Andres,C.,Bex, A.,Lehmann,J.,Zwergel,T.,Unteregg er,G.,Kamradt,J.,Stoeckle,M.and Wull ich,B.2002.Comprehensive genotypic analysis of human prostate cancer cell lines and sublines derived from metastases after orthotopic implantation in nud mice. Int.J.onc. 21:695-706.

22- König ,J.J,Wilma,T.,Jan,W.Van D., Johannes, C. R., Anne, H. and Fritz, H.S.2006.Loss and gain of chromosomes 1,18, and $\mathrm{Y}$ in prostate cancer.The Prostate 25(6):281- 291.

23- Maniatis,T.,Fritsch,E.F.and Sambrook,J.1982.Molecular cloning.A laboratory manual.Cold Spring harbor Laboratory.New York.

24- Saiki,R.K.(1988). The design \& optimization of the PCR.In:"PCR Technology Principles and Applications for DNA amplification edt.Erlich,H.A.Stockton Press,New York.

25- Hoglund,M.,Gisselsson,D.,Hansen, G.G.,Sall,T.and Mitelman,F.2002. Multivariate analysis of chromosomal inbalances in breast cancer delineates cytogenetic pathways and reveals complex relationships among inbalances.CancerRes.62:2675-2680

26- Hoglund,M.mSall,T.,Mitelman,F.,M andahl,N.and Elmula,I.F.2001.Identification of cytogenetic subgroups and karyotypic pathways in transitional cell carcinoma. Cancer Res. 61:8246-8246. 
42- .Pellman,D.2007. Cell biology:Aneuploidy and cancer.Nature 446:38-39.

43- Ruíz-Godoy,L.M.R.,Garcia-Cuellar,

C.M.,ález,N.E.,Suchil,B.L.,érez-

Cárdenas,E.,ácnchez-Pérez,Y,Yárez-

Roa,M.L.and Meneses.A.2006.Muta-

tional analysis of K-ras and Ras protein expression in larynx squamous cell carcinoma.Exp.Clin.Cancer Res. 25:73-8.

44- Shchors,K.and Evan,G. 2007.Tumor Angiogenesis: Cause or Consequence of Cancer?Cancer Res:67:7059-7061 .6.Nelson,N.J.1999. Viruses and cancer Journal of the National cancer Institute, 91, No.20,1709.

45- Shu,X.O.,Perentesis,J.P,Wen,W,Buckley,J.D., Boyle ,E., Ross ,J.A., and Robison,L.L.2004.Parental Exposure to Medications and Hydrocarbons and ras Mutations in Children with Acute Lymphoblastic Leukemia:A Report from the Children's Oncology Group .Cancer Epidemiol.Biomarkers Prev. 13 (7): 1230-1235.

46- Przybojewska,B.,Jagiellob,A.and Jalmuznac,P.2000.H-RAS,K-RAS,\&

N-RAS Gene Activation in Human Bladder Cancers.Cancer genetic and cytogenetics.121:73-77.

47- Tsao,H.,Xue,Z.,Kianna,F.and Frank, G.H.,2000.Relative Reciprocity of NRAS and PTEN/MMAC1 Alterations in Cutaneous Melanoma Cell Lines.Cancer Research 60,1800-1804.
36- Thomas,G.A.,Raffel,C.1991.Loss of heterozygosity on $6 \mathrm{q}, 16 \mathrm{q}$, and $17 \mathrm{p}$ in human central nervous system primitive neuroectodermal tumors .Cancer Res. 51:639.

37- VonDeimling,A.,Bende,B.,Jahnke,R., Waha,A.,Kraus,J.,Albrecht,S.,Wellenr euther,R.,Fassbender,F.,Nagel,J.,Menon,A.G.,Louis,D.N.,Lenartz,D.,Schramm ,J.,Wiestler,O.D.1994.Lociassociated with malignant progression in astrocytomas:a candidate on chromosome 19q. Cancer Res.54:1397.

38- Von Deimling, A.,Louis,D.N.,von Ammon,K.,Petersen,I.,Hoell,T.,Chung ,R.Y.,Martuza,R.,Schoenfeld,D.,Yasar gil,M.G.,Wiestler,O.D.,Seizinger,B.R. 1992.Association of epidermal growth factor receptor gene amplification with loss of chromosome 10 in human glioblastoma multiforme.J. Neurosurg. 77:295.

39- Fults,D.,Pedone,C.1993.Deletion mapping of the long arm of chromosome 10in glioblastoma multiforme.Genes .Chrom osom. Cancer 7:173.

40- Ruttledge,M.H.,Xie,Y.G.,Han,F.Y, Peyrard,M.,Collins,V.P,Nordenskjold, M., Dumanski,J.P.1994.Deletions on chromosome 22 in sporadic meningioma.Genes Chromosom Cancer 10:122.

41- Anwar,K.,Nakakuki,K.,Naiki,H. and Inuzuka,M.1993.ras gene mutations and HPV infection are common in human laryngeal carcinoma.Int J.Cancer.2:53(1):22-8.

\title{
Chromosomal aberrations and N-ras activation in human larynx carcinoma cell line Hep-2.
}

\begin{abstract}
In the present study, cytogenetic and molecular techniques were conducted to detect the chromosomal aneuploidy and the involvement of $\mathrm{N}$ and $\mathrm{H}$ genes in squamous larynx carcinoma cell line Hep-2.Our results showed that numerical and structural abnormalities were involved in larynx cancer Hep-2.The total number of chromosomes ranging from tripolyploidy in passage 187to more than that in passage207. The more frequent chromosomes involved in numerical aberrations were chromosomes1,7,16,17 and 18. Structural chromosomal aberrations were also detected.Deletion of short arm was detected in chromosome 1(del 1p) and the long arm of chromosome 1(del 1q)and 6(del 6q).Gaining on short arms were also recorded in chromosomes 3(3p+) and 12(12p+).At the molecular level,one allele of $\mathrm{N}$-ras proto-oncogene was found deleted in the location 61 in passage 187 and complete deletion of both locations in passage 207.These findings reflex a great genomic instability in the the tumor model used in this study. Also the results confirmed the multistages theory in cancer arising.
\end{abstract}

Abdul Hussain M. Al-Faisal, Amal M.Ali, and Nahi Y.Yassen 